\title{
Perfil Epidemiológico da Hanseníase no Município de Castanhal - Pará no período de 2014 a 2017
}

Epidemiological profile of leprosy in the municipality of Castanhal - Pará from 2014 to 2017

Perfil Epidemiológico de la Hanseniasis en el Municipio de Castanhal - Pará en el período de 2014 a 2017

Daniela Valente Cunha ${ }^{1 *}$, Emanuela Barbosa Rodrigues ${ }^{2}$, Hévila Alexandre Lameira ${ }^{3}$, Marta Thaise Smith da $\mathrm{Cruz}^{3}$, Sara Morais Rodrigues ${ }^{4}$, Fabiola da Silva dos Santos ${ }^{5}$.

\begin{abstract}
RESUMO
Objetivo: O objetivo do trabalho foi analisar dados epidemiológicos da prevalência de hanseníase do Sistema de Informação de Agravos e Notificação (SINAN), no período de 2014 a 2017. Metodologia: Para a análise dos dados, foram tabulados gráficos no programa Excel para melhor representar as variáveis: Prevalência, localidade, sexo, idade, a escolaridade, bairros acometidos e tipos de saída. Resultados: Foram registrados 224 casos de hanseníase, sendo $51,34 \%$ do sexo masculino e $48,66 \%$ do sexo feminino. A faixa etária mais acometida foi a economicamente ativa de até 40 anos e escolaridade predominantemente baixa e quanto ao tipo de saída, observou-se que mais da metade dos pacientes obtiveram a cura. Conclusão: Os resultados demonstraram que faz-se necessário uma ação intensiva de esclarecimento sobre a doença para que toda a população saiba como ocorre a transmissão, tratamento e prevenção da mesma, diminuindo assim, o preconceito e, elevando a busca pelo tratamento adequado.
\end{abstract}

Palavras Chave: Hanseníase, Prevalência, Patogenicidade.

\begin{abstract}
Objective: The objective of this study was to analyze epidemiological data on the prevalence of leprosy in the Aging and Notification Information System (SINAN), from 2014 to 2017. Methodology: For the analysis of the data, graphs were tabulated in the excel program to better represent the variables: Prevalence, location, gender, age, schooling, affected neighborhoods, and types of exit. Results: There were 224 cases of leprosy, $51,34 \%$ male and $48 \%, 66 \%$ female. The most affected age group was the economically active group of up to 40 years and schooling was predominantly low. Regarding the type of output, it was observed that more than half of the patients were cured. Conclusion: The results showed that an intensive action of enlightenment on the disease is necessary so that the entire population knows how the transmission, treatment and prevention of the disease occur, thus reducing prejudice and, thus, increasing the search for adequate treatment.
\end{abstract}

Key words: Leprosy, Prevalence, Pathogenicity.

\footnotetext{
${ }^{1}$ Escola Superior da Amazônia (ESAMAZ). São Miguel do Guamá-PA.

*E-mail: daniela valente@ymail.com

Escola Superior da Amazônia (ESAMAZ). Capanema-PA.

${ }^{3}$ Escola Superior da Amazônia (ESAMAZ). Castanhal-PA.

${ }^{4}$ Escola Superior da Amazônia (ESAMAZ). Belém-PA.

${ }^{5}$ Universidade Federal do Pará (UFPA). Belém-PA.
}

SUBMETIDO EM: 4/2019

ACEITO EM: 5/2019

PUBLICADO EM: 8/2019

REAS/EJCH | Vol.11(15) | e858 | DOI: https://doi.org/10.25248/reas.e858.2019 Página 1 de 8 


\section{RESUMEN}

Objetivo: El objetivo del trabajo fue analizar datos epidemiológicos de la prevalencia de hanseniasis del Sistema de Información de Agravios y Notificación (SINAN), en el período de 2014 a 2017. Metodología: Para el análisis de los datos, fueron tabulados gráficos en el programa excel para representar mejor las variables: Prevalencia, localidad, sexo, edad, escolaridad, barrios acometidos y tipos de salida. Resultados: Se registraron 224 casos de lepra, siendo $51,34 \%$ del sexo masculino y $48,66 \%$ del sexo femenino. El grupo de edad más acometida fue la económicamente activa de hasta 40 años y escolaridad predominantemente baja y en cuanto al tipo de salida, se observó que más de la mitad de los pacientes obtuvieron la cura. Conclusión: Los resultados demostraron que se hace necesaria una acción intensiva de esclarecimiento sobre la enfermedad para que toda la población sepa cómo ocurre la transmisión, tratamiento y prevención de la misma, disminuyendo así el prejuicio y, elevando la búsqueda por el tratamiento adecuado.

Palabras clave: Lepra, Prevalencia, Patogenicidad.

\section{INTRODUÇÃO}

A hanseníase trata-se de uma enfermidade infectocontagiosa causada por uma bactéria de evolução crônica. Segundo a Organização Mundial da Saúde (OMS), em 2016 o Brasil notificou 25.218 casos novos, totalizando uma taxa de detecção de 12,2/100 mil habitantes. O Brasil ocupa o segundo lugar mundial de notificações de casos de hanseníase, sendo uma doença de notificação compulsória no país, incluída como uma das doenças de saúde pública postadas pelo Ministério da Saúde, devido à sua alta endemicidade (BRASIL, 2018; ALVES CJMA, et al., 2010).

O período de incubação da doença varia de 2 a 7 anos, possui alta taxa de infecção, porém com baixa virulência. Sua principal característica é a manifestação de lesões cutâneas acompanhadas de diminuição de sensibilidade térmica, dolorosa e tátil. O Mal de Hansen tem como agente etiológico a bactéria Mycobacterium leprae, responsável pelo acometimento das células cutâneas e nervosas periféricas (ALVES CJMA, et al., 2010).

Desde a antiguidade, a hanseníase, antes conhecida como lepra, tem feito parte do contexto social da humanidade, sendo, portando, uma doença que ultrapassa gerações. De acordo com a Lei ํo 9010 (Diário Oficial de 30/03/1995), o termo hanseníase foi adotado oficialmente no Brasil em 1976. O nome da doença foi modificado para que houvesse a diminuição do estigma causado no passado que excluía os indivíduos afetados do convívio social (AGUIAR ZN e RIBEIRO MCS, 2006).

A doença pode levar ao desenvolvimento de surtos reacionais, episódios inflamatórios agudos e subagudos de hipersensibilidade, acometendo vários órgãos como olhos, rins, glândulas supra-renais, testículos, fígado e baço. Podendo ainda ocasionar deformidades nos olhos, mãos e pés no decorrer e evolução da manifestação (EIDT LM, 2004; MEDINA NH et al., 2004).

A Hanseníase tem sido descrita no mundo há anos, seus primeiros relatos foram ocorreram no Egito, na Índia, China e Japão a 4.300 anos a.C. (BRASIL, 1960). No final do século XIX esta enfermidade foi identificada pelo médico norueguês Gerhard Henrik Armauer Hansen que a partir de estudos observacionais detectou o agente causador desta patologia (GOMES ACB, 2000; SANTOS FSD, et al., 2008). A primeira documentação comprovatória de Hanseníase no Brasil data de 4 de dezembro de 1697, no estado do Rio de Janeiro (OPROMOLLA PA e LAURENTI R, 2011).

De acordo com a taxonomia de Mycobacterium leprae este pertence a ordem Actinomycelalis e família Mycobaderiaceae. Em relação a sua morfologia, possui a forma de bacilo reto ou levemente arqueado, com extremidades arredondadas, seu tamanho varia de 1 a $8 \mathrm{~mm}$ de comprimento e $0,3 \mathrm{~mm}$ de diâmetro apresenta-se, como intracelular obrigatório e infecta com maior frequência macrófagos (OPROMOLLA DVA, et al., 2000). 
Os nervos periféricos são os mais acometidos pelo bacilo, tendo preferência pela pele e células de Schwann, responsável pela produção de mielina que envolvem os axônios, ocasionando a maior parte das complicações. Os olhos e as vias aéreas superiores também são afetados causando danos irreparáveis da função dos nervos como degeneração axonal, fibrose aumentada, desmielinização e deficiência crônica (PEREIRA DL, et al., 2012).

A transmissão ocorre através do contato direto com a pessoa enferma e o principal meio de contágio se dá por vias respiratórias através de gotículas (tosse ou espirro). A hanseníase tem o homem como o portador do bacilo. Essa patologia acomete pessoas de diversas idades e ambos os sexos e pode suceder em um longo prazo, durando em média de 2 a 7 anos de incubação. Outro meio de transmissão da doença está relacionado com as condições precárias do indivíduo e o convívio com pessoas em um mesmo espaço, o que pode influenciar no risco de contrair a doença (BRASIL, 2002). Segundo Pereira DL, et al. (2012), lesões cutâneas podem expelir numerosos bacilos e ferimentos na pele sendo capaz de servir de porta de entrada para essa microbactéria.

De acordo com o Ministério da Saúde, os principais sinais e sintomas da hanseníase são: manchas esbranquiçadas (hipocrômicas), acastanhadas ou avermelhadas na pele, com alterações de sensibilidade ao calor e/ou ao tato, formigamentos, choques e câimbras nos braços e pernas, que evoluem para dormência - a pessoa se queima ou se machuca sem perceber, pápulas, tubérculos e nódulos (caroços), normalmente sem sintomas, diminuição ou queda de pelos, localizada ou difusa, especialmente nas sobrancelhas, pele avermelhada, com diminuição ou ausência de suor no local (BRASIL, 2017).

Além dos sinais e sintomas mencionados, pode-se observar: dor, choque e/ou espessamento de nervos periféricos, diminuição e/ou perda de sensibilidade nas áreas dos nervos afetados, principalmente nos olhos, mãos e pés, diminuição e/ou perda de força nos músculos inervados por estes nervos, principalmente nos membros superiores e inferiores, edema de mãos e pés com cianose (arroxeamento dos dedos) e ressecamento da pele, febre e artralgia, associados a caroços dolorosos, de aparecimento súbito, surgimento repentino de manchas dormentes com dor nos nervos dos cotovelos, joelhos e tornozelos, entupimento, feridas e ressecamento do nariz, sensação de secura e areia nos olhos (BRASIL, 2002).

O diagnóstico da hanseníase é baseado na resposta inflamatória da doença. Primeiramente, deve-se fazer anamnese seguido de uma avaliação dermatológica, onde resulta na identificação de lesões de pele com alteração de sensibilidade, e uma neurológica no qual são identificados neurites e deformidades que ajudam a categorizar o nível de incapacidade física (BRASIL, 2002).

No diagnóstico dermatológico a doença é diferenciada baseada de acordo com o número das lesões na pele, o que dará a classificação operacional da doença. Até cinco lesões são classificadas em Paucibacilar (PB), apresentando mais de cinco lesões é considerada a forma Multibacilar (MB) (NEVES DCO, et al. ,2017). De acordo com a classificação será direcionado ao paciente o esquema do tratamento com Poliquimioterápicos (BRASIL, 2016).

A forma Paucibacilar é considerada menos grave, possuindo dois tipos, Hanseníase Tuberculoide e Indeterminada, que se manifestam por meio de lesões hipopigmentadas e anestésicas. A forma Multibacilar é subdividida em Hanseníase Dimorfa que geralmente é caracterizada por manifestar manchas avermelhadas ou esbranquiçadas com bordas elevadas e hanseníase Virchowiana, esta apresenta-se como a forma mais contagiosa, a pessoa acometida não apresenta manchas visíveis, porém a pele apresenta-se seca, avermelhada e infiltrada com os poros expandidos (PEREIRA DL, et al., 2012; BRASIL, 2017). No diagnóstico laboratorial é realizada a baciloscopia, onde é analisado o Mycobacterium leprae, através do esfregaço de raspados das lesões cutâneas ou de outras áreas afetadas como, lóbulos auriculares e/ou cotovelos. É fundamental selecionar a área que haja linfa, para que a mesma seja coletada e posicionada sobre a lâmina para, em seguida, ser realizada a coloração adequada para melhor visualização microscópica. Há doenças que ocasionam sintomas semelhantes de caracterização da hanseníase, como doenças de pele e neurológicas, consequentemente se faz necessário o diagnóstico diferencial (BRASIL, 2002). 
Em 1980, a OMS (Organização Mundial da Saúde) passou a indicar a poliquimioterapia (PQT) para o tratamento da hanseníase (EIDT LM, 2004). A PQT é a associação de Rifampicina, Dapsona e Clofazimina, o esquema terapêutico é feito conforme as formas clínicas apresentadas. Para pacientes paucibacilares (PB) a medicação é feita durante seis meses com Rifampicina e Dapsona, e para pessoas com a forma multibacilar (MB) a duração do tratamento é de doze meses, com Rifampicina, Dapsona e Clofazimina (GODINHO BVP, et al., 2014).

Segundo o Ministério da Saúde, não há um meio específico de profilaxia para hanseníase, dessa forma, a mesma deve ser prevenida por meio do diagnóstico precoce de casos, de educação em saúde acerca da doença, tratamento eficiente dos doentes e a utilização do Bacillus Calmette-Guérin, vacina utilizada para a prevenção de tuberculose que também pode ter efeito sobre a hanseníase (BRASIL, 2000 e 2016).

O Norte está entre as principais regiões brasileiras que apresentam altas taxas de prevalência concentrando a maior parte dos casos confirmados (EIDT LM, 2004). Segundo o Ministério da Saúde, o Pará foi o quarto em número de pessoas com hanseníase em 2015, apresentando a taxa de detecção geral de novos casos em 35, 2/ 100 mil habitantes (NEVES DCO, et al., 2017). Segundo uma pesquisa realizada por Lima RAA e Barreto JG (2011), no município de Castanhal no período de 2004 a 2010, o município foi considerado hiperendêmico por apresentar taxa de detecção anual de casos novos em 2009 de 48,2/100.000 habitantes, tendo sidos notificados 633 casos no período do estudo.

Em virtude dos fatos mencionados e da necessidade de pesquisas nesta área, o objetivo desde artigo é analisar dados epidemiológicos da prevalência de hanseníase do Setor de Vigilância Epidemiológica do município de Castanhal, Pará no período de 2014 a 2017.

\section{MÉTODOS}

O presente estudo aborda uma pesquisa descritiva epidemiológica de análise quantitativa. Os dados foram coletados das fichas do Sistema de Informação de Agravos de Notificação (SINAN), cedidos pelo departamento de Vigilância Epidemiológica da Secretaria Municipal de Castanhal. As informações analisadas foram referentes à incidência de casos de hanseníase no referido município no período de 2014 a 2017. O presente trabalho dispensa o uso do Termo de Consentimento Livre e Esclarecido por não fazer uso de amostras biológicas, sendo utilizados somente dados coletos em fichas epidemiológicas.

Com área de 1. 028, $889 \mathrm{~km}^{2}$ e número populacional de 195.253 (IBGE, 2017), Castanhal fica a $65 \mathrm{~km}$ de Belém - PA, está entre as cinco principais cidades do Estado e figura como uma espécie de metrópole da região Nordeste do Pará (Prefeitura Municipal de Castanhal, 2018).

Para a análise dos dados, foram tabulados por meio do programa Microsoft Excel / 2010 gráfico e tabelas com as seguintes variáveis: Prevalência, sexo, idade, escolaridade, bairros acometidos e tipos de saída. Mediante a análise de tais dados, foram construídos os resultados, discussão e conclusão. Alguns dados foram desconsiderados por não constarem na tabela cedida pelo Sistema de Informação de Agravos e Notificação (SINAN) o que ocasionou alteração nos valores.

\section{RESULTADOS E DISCUSSÃO}

Segundo os dados do Sistema de Informação de Agravos de Notificação (SINAN) foram registrados 224 casos de hanseníase no período de 2014 a 2017 no município de Castanhal-Pará. O ano de 2014 foi o que apresentou maior taxa de prevalência, totalizando 73 casos (33\%), e o ano de 2015, foi o que apresentou menor ocorrência de casos de hanseníase, totalizando apenas 40 casos (18\%) (Gráfico 1).

Ao analisar os dados, foi possível detectar oscilações significativas, principalmente, do ano de 2014 para o ano de 2015, isso pode ser explicado por possíveis ações de controle da doença no município. Vale ressaltar que a ocorrência de casos detectados em uma região pode ser influenciada por ações educativas aplicadas no local e a capacitação dos profissionais de saúde para realizarem o diagnóstico da doença de maneira eficiente, competente, adequada e precoce (Organização Mundial da Saúde, 2006). Há registros de 
uma Ação da Sociedade Brasileira de Hansenologia no município de Castanhal-PA no ano de 2016, no qual houve a aplicação de curso teórico-prático para profissionais de saúde envolvendo estudantes e vários profissionais qualificados (Sociedade Brasileira de Hansenologia - SBH, 2016).

Gráfico 1 - Prevalência de casos de Hanseníase no período de 2014 a 2017, no município de Castanhal, Pará.

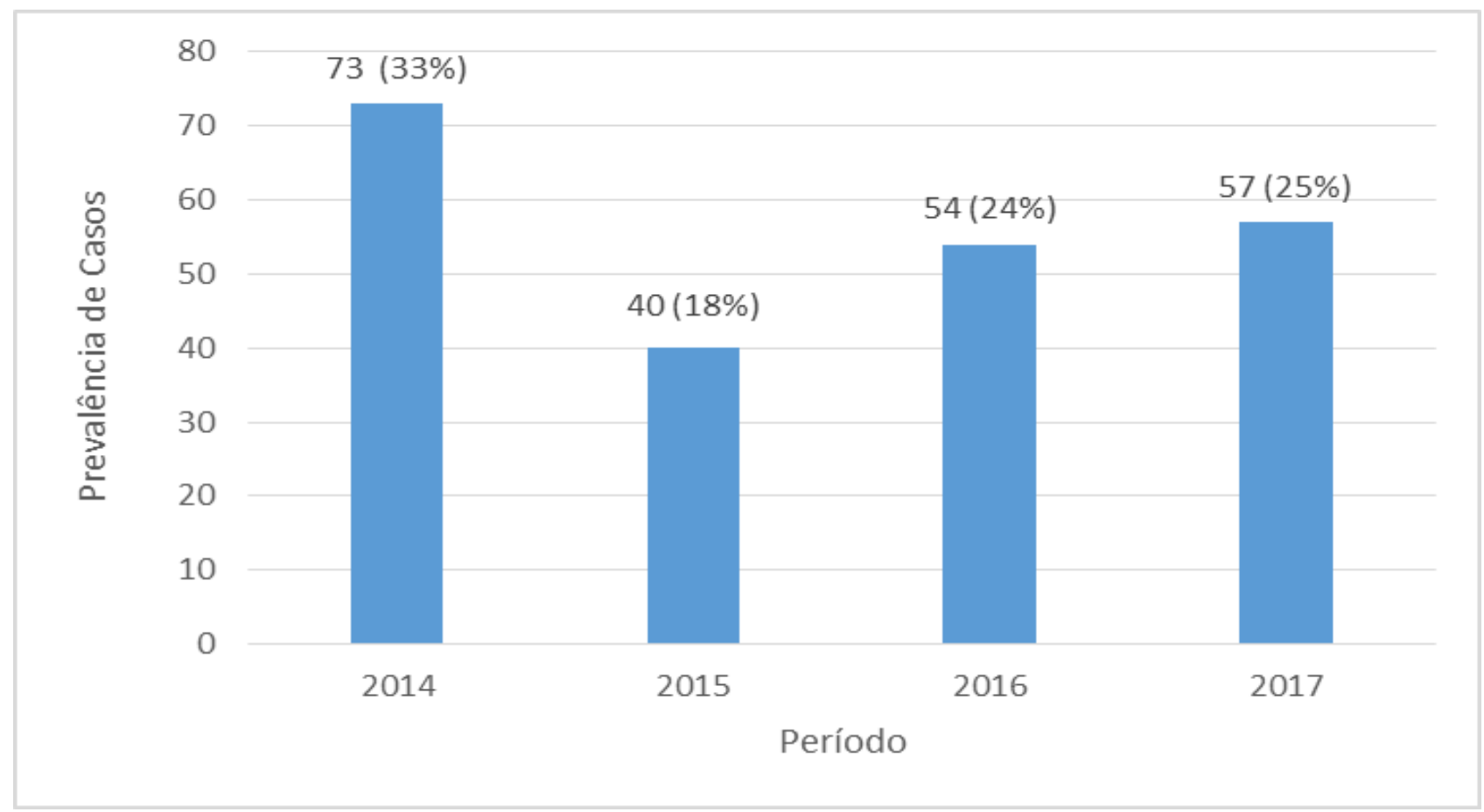

Fonte: Sistema de Informação de Agravos e Notificação (SINAN) /2018.

Em relação a variável de gênero, embora a diferença tenha sido pequena, foi possível observar que ocorreu maior prevalência no sexo masculino $(51,34 \%)$ em relação ao sexo feminino $(48,66 \%)$. Houve maior número de ocorrências de hanseníase na população masculina em 2014, sendo representadas por 18,30\% dos casos $(\mathrm{N}=41)$ e em 2016 a população feminina apresentou $14,29 \%(\mathrm{~N}=32)$ dos casos registrados (Tabela 1).

O predomínio da hanseníase em homens também foi relatado no Boletim Epidemiológico do Ministério da Saúde entre os anos de 2012 a 2016. Segundo Melão S, et al. (2011), o aumento do número de casos em indivíduos do sexo masculino pode ser explicado pelo fato de estes apresentarem maior exposição a ambientes de risco, além disso, o fato dos homens, de maneira geral, apresentarem menor preocupação em relação a estética acaba dificultando o diagnóstico (SILVA AR, et al., 2010). Por outro lado, Lana FCF, et al., (2003) e Santos AD, et al., (2016) obtiveram resultados contrários em seus estudos, em que as mulheres apresentaram maior prevalência da hanseníase em relação aos homens, nos estados de Minas Gerais entre os anos de 1990 e 2000 e Bahia no período de 2002 a 2012, respectivamente.

Em relação a prevalência de casos de Hanseníase por faixa etária de 2014 a 2017. No ano de 2014, a faixa etária mais acometida foi até 20 anos (8,03\%) e as menos acometidas foram entre 51 a 60 e 61 a 70 anos de idade (2,67\%). Em 2015, a maior prevalência ocorreu entre 31 a 40 anos (4,91\%), já a menor foi em idosos com mais de 70 anos $(0,89 \%)$. No ano posterior, em 2016, o maior número de casos foi dividido em duas faixas etárias, até 20 anos e dos 31 a 40 anos de idade (6,69 \% para ambas) e o menor número de casos refere-se a pacientes a partir de 61 anos de idade (0,89\%). O ano de 2017 apresentou o maior índice de casos em indivíduos de 21 a 30 anos (4,91\%) e o menor índice está dividido em três faixas de idade, 41 a 50 anos (3,12\%), 51 a 60 anos (3,12\%) e maiores de 70 anos $(3,12 \%)$. 
Tabela 1 - Prevalência de casos de Hanseníase com dados sociodemográficos no período de 2014 a 2017, no município de Castanhal/Pará.

\begin{tabular}{|c|c|c|c|c|c|c|c|c|c|c|}
\hline \multirow[b]{2}{*}{ VARIÁVEIS } & \multicolumn{2}{|c|}{2014} & \multicolumn{2}{|c|}{2015} & \multicolumn{2}{|c|}{2016} & \multicolumn{2}{|c|}{2017} & \multicolumn{2}{|c|}{ TOTAL } \\
\hline & $\mathbf{N}$ & $\%$ & $\mathbf{N}$ & $\%$ & $\mathbf{N}$ & $\%$ & $\mathbf{N}$ & $\%$ & $\overline{\mathbf{N}}$ & $\%$ \\
\hline \multicolumn{11}{|l|}{ SEXO } \\
\hline Masculino & 41 & $18,30 \%$ & 19 & $8,48 \%$ & 22 & $9,82 \%$ & 33 & $14,73 \%$ & 115 & $51,34 \%$ \\
\hline Feminino & 32 & $14,29 \%$ & 21 & $9,38 \%$ & 32 & $14,29 \%$ & 24 & $10,71 \%$ & 109 & $48,66 \%$ \\
\hline \multicolumn{11}{|l|}{ FAIXA ETÁRIA } \\
\hline Até 20 anos & 18 & $8,03 \%$ & 4 & $1,78 \%$ & 15 & $6,69 \%$ & 8 & $3,57 \%$ & 45 & $20,07 \%$ \\
\hline 21-30 anos & 13 & $5,80 \%$ & 9 & $4,01 \%$ & 7 & $3,12 \%$ & 11 & $4,91 \%$ & 38 & $17,84 \%$ \\
\hline 31-40 anos & 9 & $4,01 \%$ & 11 & $4,91 \%$ & 15 & $6,69 \%$ & 9 & $4,01 \%$ & 41 & $19,62 \%$ \\
\hline $41-50$ anos & 11 & $4,91 \%$ & 4 & $1,78 \%$ & 6 & $2,67 \%$ & 7 & $3,12 \%$ & 28 & $12,48 \%$ \\
\hline 51- 60 anos & 9 & $2,67 \%$ & 6 & $2,67 \%$ & 7 & $3,12 \%$ & 7 & $3,12 \%$ & 29 & $11,58 \%$ \\
\hline $61-70$ anos & 6 & $2,67 \%$ & 4 & $1,78 \%$ & 2 & $0,89 \%$ & 8 & $3,57 \%$ & 20 & $8,91 \%$ \\
\hline Mais de 70 anos & 7 & $3,12 \%$ & 2 & $0,89 \%$ & 2 & $0,89 \%$ & 7 & $3,12 \%$ & 18 & $8,02 \%$ \\
\hline
\end{tabular}

Fonte: Sistema de Informação de Agravos e Notificação (SINAN) /2018.

A partir deste resultado, é possível inferir que a população que mais sofre com a hanseníase é a economicamente ativa, mais especificamente até 40 anos, o que pode interferir de maneira significativa na economia do município, haja vista que os indivíduos dessa faixa etária podem sofrer de males como incompetências, lesões, estados reacionais, abdicar de atividades produtivas e gerar um custo social elevado (LANA FCF, et al., 2004 e ARAUJO MG, 2003). Foram vistos fatos semelhantes em uma pesquisa realizada em Paracatu, Minas Gerais no ano de 2004 a 2006, na qual ressaltou que maior prevalência se encontra em adolescentes ( $72,1 \%$ dos casos), a falta de informação sobre a doença, acesso ao tratamento e diagnostico, e baixa renda familiar são fatores favoráveis para o desenvolvimento da doença. Vale ressaltar que na pesquisa, os adolescentes desconhecem os primeiros sintomas e por conta disso não buscam o diagnóstico, portanto o número é predominante nesta faixa etária (FERREIRA IN, et al., 2007) (Tabela 1).

Além disso, uma pesquisa feita na região Nordeste do Brasil no período de 2010 a 2014 consta que o surgimento de novos casos em menores de 15 anos vem causando preocupações para o Programa de Controle da Hanseníase, dessa forma se faz necessário que haja monitoramento da doença nessa faixa etária (CHAVES AEP, et al., 2015).

No que diz respeito à escolaridade dos indivíduos acometidos, os resultados demonstraram que o maior número de pacientes possui escolaridade entre a $5^{\mathrm{a}}$ e a $8^{\mathrm{a}}$ série incompleta do ensino fundamental, totalizando $59(26,44 \%)$ casos e, em seguida, os indivíduos que possuem escolaridade entre $1^{\text {a }}$ a $4^{4^{a}}$ série incompleta do ensino fundamental totalizando 44 casos (19,64\%) (Tabela 2).

Segundo relatos de uma pesquisa do ano de 2000 a 2006 no município de Uberaba-MG, em relação a escolaridade, afirma que $34 \%$ dos registrados tinham estudado no máximo até o 6 o ano do ensino fundamental e que este problema pode acarretar na falta de informação sobre a doença e os devidos cuidados que precisam ocorrer (MIRANZI SSC, et al., 2010).

Com relação aos bairros acometidos, observou-se que o maior número de casos foi predominante na zona urbana, no bairro Jaderlândia, com 15 casos no ano de 2014, em relação à zona rural que no mesmo ano, obteve apenas 6 casos. Relatos semelhantes foram encontrados em uma pesquisa feita no município de Mossoró-RN, entre os anos de 2004 a 2009, onde a mesma analisou o número de casos de hanseníase por zona domiciliar. A Pesquisa constatou que o índice de ocorrências na zona urbana é 6,2 vezes maior 
em relação à zona rural e que a causa dessa decorrência é a falta de infraestrutura na saúde e educação nos bairros menos favorecidos, desta forma, isto contribui significativamente para a disseminação do bacilo (SANTOS PC e SARAIVA AKM, 2010).

Ainda sobre a zona domiciliar, outro estudo feito em Rondônia no período de 2001 a 2012, constatou que o maior número dos casos analisados foi em moradores das localidades urbanas (VIEIRA GD, et al., 2014).

Quanto ao tipo de saída, observou-se que mais da metade dos pacientes obtiveram a cura, totalizando 166 casos $(74,11 \%)$ de indivíduos totalmente recuperados da enfermidade e houve apenas um óbito. Vale ressaltar, que de acordo com Pereira Junior FAC (2011), o tratamento adequado da doença é eficiente para prevenção e cura da mesma.

Tabela 2 - Distribuição dos casos mais recorrentes de Hanseníase segundo as variáveis: Escolaridade, Bairros acometidos e Tipos de saída no período de 2014 a 2017, no município de Castanhal/Pará.

\begin{tabular}{lcccccc}
\hline & \multicolumn{7}{c}{ № DE CASOS } \\
\hline ESCOLARIDADE & $\mathbf{2 0 1 4}$ & $\mathbf{2 0 1 5}$ & $\mathbf{2 0 1 6}$ & $\mathbf{2 0 1 7}$ & Total & $\%$ \\
$\mathbf{1}^{\mathrm{a}}$ a 4 (a série incompleta do EF & 14 & 11 & 6 & 13 & 44 & $\mathbf{1 9 , 6 4 \%}$ \\
$\begin{array}{l}\mathbf{5}^{\mathrm{a}} \text { a 8 } \\
\text { BAIRROS série incompleta do EF }\end{array}$ & 24 & 9 & 15 & 11 & 59 & $\mathbf{2 6 , 3 4 \%}$ \\
$\begin{array}{l}\text { Zona Urbana } \\
\text { Jaderlândia }\end{array}$ & & & & & & \\
$\begin{array}{l}\text { Zona Rural } \\
\text { Não identificado }\end{array}$ & 15 & 9 & 4 & 5 & 33 & $\mathbf{1 4 , 7 3 \%}$ \\
$\begin{array}{l}\text { TIPO DE SAÍDA } \\
\text { Cura }\end{array}$ & 6 & 4 & 5 & 5 & 20 & $\mathbf{8 , 9 3 \%}$ \\
\hline
\end{tabular}

Fonte: Sistema de Informação de Agravos e Notificação (SINAN) /2018.

Segundo Moreira AJ, et al. (2014), é preciso um trabalho articulado para que a população seja orientada sobre os sinais e sintomas da doença. Em um estudo realizado em 2012 com usuários de duas Unidades Básicas de Saúde (UBS) em Uberaba-MG, foi apontada grande significância nos resultados pós ação educativa sobre a Hanseníase abordando os tópicos: sinais e sintomas, meios de trasmissão, complicações, tratamento e prevenção, onde obtiveram retorno positivo do público alvo. Embora existam conhecimentos acerca da doença, ainda há certa rejeição, preconceito e desinformações (CORIOLANOMARINUS MWL, et al., 2012).

\section{CONCLUSÃO}

Pela observação dos aspectos analisados pôde-se traçar o perfil epidemiológico da prevalência de Hanseníase no município de Castanhal-Pará entre os anos 2014 e 2017 através dos dados cedidos pelo Setor de Vigilância Epidemiológica (Secretaria Municipal de Saúde). O Pará é o quarto Estado brasileiro mais acometido pela doença, embora a pesquisa demonstre um alto índice de cura. Foi observado um número acentuado de casos de hanseníase até o ano de 2017, o que levanta a necessidade de ações intensivas de esclarecimento sobre a doença para que toda a população saiba como ocorre a transmissão, tratamento e prevenção, diminuindo assim, o preconceito e, em contrapartida, elevar a busca pelo tratamento adequado.

\section{AGRADECIMENTOS}

A Escola Superior da Amazônia (ESAMAZ); ao Departamento de Vigilância Epidemiológica da Secretaria Municipal de Castanhal e a contribuição da nossa orientadora Mestre Professora Fabíola dos Santos. 


\section{REFERÊNCIAS}

1. AGUIAR ZN, RIBEIRO MCS. Doenças transmissíveis. 2ª Edição. São Paulo: Martinari, 2006.

2. ARAÚJO MG. Hanseníase no Brasil. Rev Soc Bras Med Trop; 36: 373-382, 2003.

3. ALVES CJMA, et al. Avaliação do grau de incapacidade dos pacientes com diagnóstico de hanseníase em Serviço de Dermatologia do Estado de São Paulo. Revista da Sociedade Brasileira de Medicina Tropical 43 (4): 460-461, jul-ago, 2010.

4. BRASIL. Ministério da Saúde. Departamento Nacional de Saúde. Serviço Nacional de Lepra. Manual de Leprologia. Rio de Janeiro: 1960.

5. BRASIL. Ministério da Saúde. Controle da hanseníase: uma proposta de integração ensino-serviço. Rio de Janeiro: DNDS/NUTES, 1989.

6. BRASIL. Ministério da Saúde. Diretrizes Nacionais para a Elaboração de Programas de Capacitação para a Equipe de Saúde da Rede Básica Atuar nas Ações de Controle de Hanseníase. Área Técnica de Dermatologia Sanitária. Brasília: 2000.

7. BRASIL. Ministério da Saúde. Secretaria de Políticas de Saúde. Departamento de Atenção Básica. Guia para o controle da hanseníase. 1aㅡ edição. Brasília, 2002.

8. BRASIL. Ministério da Saúde. Secretaria de Vigilância em Saúde - Departamento de Vigilância das Doenças Transmissíveis. Diretrizes para Vigilância, atenção e eliminação da hanseníase como problema de saúde pública. Manual técnico operacional. Brasília - Ministério da Saúde: 2016. 60 p.

9. BRASIL. Ministério da Saúde. Secretaria de Vigilância em Saúde - Departamento de Vigilância e Doenças Transmissíveis. Guia Prático Sobre a Hanseníase. Brasília: Ministério da Saúde, 2017.

10. BRASIL. Ministério da Saúde. Secretaria de Vigilância em Saúde - Boletim Epidemiológico. Caracterização da Situação Epidemiológica da Hanseníase e diferenças por sexo, Brasil, 2012-1016. Volume 49, № 4, 2018.

11. CORIOLANO-MARINUS MWL, et al. Saúde do escolar: uma abordagem educativa sobre Hanseníase. Saúde \& Transformação Social, $\quad$ ISSN 2178-7085, Florianópolis, v.3, n.1, p.72-78, 2012.

12. CHAVES AEP, et al. Hanseníase em idosos no Nordeste do Brasil. Anais CIEH, Vol. 2, N.1 ISSN 2318-0854, 2015.

13. EIDT LM. Breve história da hanseníase: sua expansão do mundo para as Américas, o Brasil e o Rio Grande do Sul e sua trajetória na saúde pública brasileira. Revista Saúde e Sociedade. №.2, v.13, p.76-88, maio-ago. 2004.

14. FERREIRA IN, et al. Distribuição espacial na população escolar em Paracatu - Minas Gerais, realizada por meio da busca ativa (2004 e 2006). Rev Bras Epidemiol, 10 (4): 555-67, 2007.

15. GODINHO BVP, et al. Hanseníase: Revisão de literatura. Brazilian Journal of Surgery and Clinical Research - BJSCR. Vol.9, n.1, pp.49-53 (Dez 2014 - Fev 2015).

16. GOMES ACB. O processo de Armauer Hansen. Jornal do Conselho Regional de Medicina do Rio Grande do Sul, p.13, fev. 2000.

17. IBGE. Dados sobre a população de Castanhal - PA, dados de 2017. Disponível em:

18. https://cidades.ibge.gov.br/brasil/pa/castanhal/panorama. Acesso em: 16 fev. 2018.

19. LANA, FCF, et al. Distribuição da Hanseníase segundo sexo no Município de Governador Valadares, Minas Gerais, Brasil. Hansenologia Internationalis, 28(2): 131-137, 2003.

20. LANA FCF, et al. Estimativa da prevalência oculta da hanseníase no Vale do Jequitinhonha: Minas Gerais. Rev Min Enferm; 8: 295-300, 2004.

21. LIMA RAA. BARRETO JG. Estudo Geoespacial da Hanseníase no município de Castanhal, Pará. ISSN 2176-1213 - Semin. de IC. da UFPA, Belém, v. 22, n.1, 2011.

22. MEDINA NH, et al. Vigilância epidemiológica das incapacidades oculares em hanseníase. Hansenologia Intemationalis, no29, v.2, p101-105, 2004.

23. MELÃO S, et al. Perfil epidemiológico dos pacientes com hanseníase no extremo sul de Santa Catarina, no período de 2001 a 2007. Revista da Sociedade Brasileira de Medicina Tropical 44(1):79-84, jan-fev, 2011.

24. MIRANZI SSC, et al. Perfil epidemiológico da hanseníase em um município brasileiro, no período de 2000 a 2006. Revista da Sociedade Brasileira de Medicina Tropical 43(1):62-67, jan-fev, 2010.

25. MOREIRA AJ, et al. Ação educativa sobre hanseníase na população usuária das unidades básicas de saúde de UberabaMG. Saúde em Debate [online].v.38, n.101, pp. 234-243. ISSN 0103-1104, 2014.

26. NEVES DCO, et al. Tendência das taxas de detecção de hanseníase em jovens de 10 a 19 anos de idade nas Regiões de Integração do estado do Pará, Brasil, no período de 2005 a 2014. Ver Pan-Amaz Saude, 2017.

27. OPROMOLLA DVA, et al. Noções de Hansenologia. Bauru: Centro de Estudos Dr. Reynaldo Quagliato, 2000.

28. OPROMOLLA PA, LAURENTI R. Controle da Hanseníase no Estado de São Paulo: análise histórica. Revista Saúde Pública 45(1):195-203. São Paulo-SP, 2011.

29. Organização Mundial da Saúde. Estratégia global aprimorada para redução adicional da carga da hanseníase: período do plano: 2011-2015. Brasília: Organização Mundial da Saúde; 2006.

30. PEREIRA JUNIOR FAC. Motivos do abandono ou interrupção do tratamento da hanseníase: uma revisão sistemática da literatura. 2011. Monografia (Gestão de saúde coletiva) - Fundação Oswaldo Cruz. 2011.

31. PERERIA DL, et al. Estudo da Prevalência das Formas Clínicas da Hanseníase na Cidade de Anápolis-Go. Ensaios e Ciência: Ciências Biológicas, Agrárias e da Saúde.Vol. 16, №. 1, 2012.

32. Prefeitura Municipal de Castanhal. 2018. In: Institucional. Sobre Castanhal. Disponível em:

33. http://www.castanhal.pa.gov.br/institucional/\#SobreCastanhal. Acesso em: 16 fev. 2018.

34. SANTOS FSD. Óleo de Chaulmoogra como conhecimento científico: a construção de uma terapêutica antileprótica. História, Ciências, Saúde - Manguinhos, Rio de Janeiro. v.15, n.1, p.29-47, jan.-mar, 2008.

35. SANTOS PC, SARAIVA AKM. Panorama dos casos de hanseníase na zona urbana e rural do município de Mossoró entre os anos de 2004 e 2009. 62 ${ }^{\mathrm{a}}$ Reunião Anual da SBPC, 2010.

36. SANTOS AD, et al. Análise espacial e características epidemiológicas dos casos de hanseníase em área endêmica. Rev enferm UFPE online. Recife, 10(Supl. 5):4188-97, nov. 2016.

37. SILVA AR, et al. Hanseníase no município de Buriticupu, estado do Maranhão: busca ativa de casos na populaçãoo adulta. Revista da Sociedade Brasileira de Medicina Tropical 43(6):691-694, nov-dez, 2010.

38. AÇÃO CASTANHAL (PA). 2016. In: Sociedade Brasileira de Hansenologia - SBH. Disponível em: http://www.sbhansenologia.org.br/acao/16-08-2016-acao-castanhal-pa. Acessado em: 15 jul. 2019.

39. VIEIRA GD, et al. Hanseníase em Rondônia: incidência e características dos casos notificados, 2001 a 2012. Epidemiologia. Serv. Saúde v.23 n.2 Brasília jun. 2014. 